\title{
A role for the DP subunit of the E2F transcription factor in axis determination
} \section{during Drosophila oogenesis}

\author{
Denise L. Myster1,2, Peter C. Bonnette1,3 and Robert J. Duronio1,2,3,4,* \\ ${ }^{1}$ Department of Biology, ${ }^{2}$ Lineberger Comprehensive Cancer Center, ${ }^{3}$ Program in Molecular Biology and Biotechnology, and \\ ${ }^{4}$ Curriculum in Genetics and Molecular Biology, University of North Carolina, Chapel Hill, NC 27599, USA \\ *Author for correspondence at address 1 (e-mail: duronio@med.unc.edu) \\ Accepted 18 May; published on WWW 10 July 2000
}

\section{SUMMARY}

The E2F family of transcription factors contributes to cell cycle control by regulating the transcription of DNA replication factors. Functional ' $\mathrm{E} 2 \mathrm{~F}$ ' is a DNA-binding heterodimer composed of E2F and DP proteins. Drosophila contains two E2F genes $(d E 2 F, d E 2 F 2)$ and one DP gene $(d D P)$. Mutation of either $d E 2 F$ or $d D P$ eliminates G1-S transcription of known replication factors during embryogenesis and compromises DNA replication. However, the analysis of these mutant phenotypes is complicated by the perdurance of maternally supplied gene function. To address this and to further analyze the role of E2F transcription factors in development we have phenotypically characterized mitotic clones of $d D P$ mutant cells in the female germline. Our analysis indicates that dDP is required for several essential processes during oogenesis. In a fraction of the mutant egg chambers the germ cells execute one extra round of mitosis, suggesting that in this tissue dDP is uniquely utilized for cell cycle arrest rather than cell cycle progression. Mutation of $d D P$ in the germline also prevents nurse cell cytoplasm transfer to the oocyte, resulting in a 'dumpless' phenotype that blocks oocyte development. This phenotype likely results from both disruption of the actin cytoskeleton and a failure of nurse cell apoptosis, each of which are required for normal cytoplasmic transfer. Lastly, we found that dDP is required for the establishment of the dorsal-ventral axis, as loss of dDP function prevents the localized expression of the EGFR ligand Gurken in the oocyte, which initiates dorsalventral polarity in the egg chamber. Thus we have uncovered new functions for E2F transcription factors during development, including an unexpected role in pattern formation.

Key words: E2F, Drosophila, Oogenesis, Gurken, Cell cycle, Axis determination

\section{INTRODUCTION}

Control of the cell cycle is essential for the development of an organism. E2F transcription factors play a key role in the control of cell proliferation by imparting both positive and negative influences on progression through the cell division cycle (reviewed in Dyson, 1998). In its positive role, E2F activates the transcription of a broad spectrum of genes required for cell cycle progression (e.g. cyclin $E$ and $C D C 25 A$ ) and DNA synthesis (e.g. DHFR, RNR) (DeGregori et al., 1997; Vigo et al., 1999). In its negative role, $\mathrm{E} 2 \mathrm{~F}$ is converted into a transcriptional repressor by binding to a member of the $\mathrm{pRb}$ family of 'pocket proteins'. The association of pocket proteins with E2F is controlled in large part by cyclin/cyclin-dependent-kinase (cdk) mediated phosphorylation, with hypophosphorylated forms of the pocket proteins preferentially binding E2F during the $\mathrm{G}_{1} / \mathrm{G}_{0}$ phase of the cell cycle. Pocket protein mediated repression is relieved by $\mathrm{G}_{1}$ cyclin/cdk (i.e. cyclin D/cdk4 and cyclin E/cdk2) dependent phosphorylation in response to growth stimulation, causing entry into the cell cycle (Harbour et al., 1999). Several studies using $E 2 F$ knockout alleles in mice are consistent with this idea that E2F has properties of both an inhibitor and a promoter of cell proliferation in vivo (Field et al., 1996; Humbert et al., 2000; McCaffrey et al., 1999; Pan et al., 1998; Tsai et al., 1998; Yamasaki et al., 1998, 1996).

Genetic experiments in Drosophila are also consistent with this paradigm of E2F function. E2F is a transcriptional heterodimer with one subunit encoded by the $E 2 F$ family of genes and one by the $D P$ family. Two E2F ( $d E 2 F$ and $d E 2 F 2)$ genes, one DP $(d D P)$ gene, and one pocket protein $(R B F)$ gene have been reported in flies (Du et al., 1996; Dynlacht et al., 1994; Hao et al., 1995; Ohtani and Nevins, 1994; Sawado et al., 1998). Loss-of-function mutations of $d E 2 F, d D P$ and $R B F$ indicate that each is an essential gene required for normal Drosophila development (Du and Dyson, 1999; Duronio et al., 1998, 1995; Royzman et al., 1997). Both $d E 2 F$ and $d D P$ mutant embryos fail to activate the expression of genes involved in DNA replication (e.g. cyclin $E, R N R 2$ and $O R C 1$ ) at the $\mathrm{G}_{1}-\mathrm{S}$ transition (Asano and Wharton, 1999; Duronio et al., 1998, 1995; Duronio and O'Farrell, 1995; Royzman et al., 1997), and E2F binding sites confer activation of reporter genes in transgenic flies (Asano and Wharton, 1999; Yamaguchi et al., $1997,1995)$. DNA synthesis in $d E 2 F$ and $d D P$ mutant embryos is attenuated (Duronio et al., 1998; Royzman et al., 1997), and 
clones of $d E 2 F$ mutant cells in wing and eye imaginal tissue grow very slowly and are eliminated due to competition with neighboring wild-type cells (Brook et al., 1996; Neufeld et al., 1998). These data are consistent with the model that transactivation by E2F stimulates cell cycle progression. Conversely, RBF/dE2F/dDP complexes appear to be important for downregulating transcription during cell cycle arrest. RBF prevents $\mathrm{dE} 2 \mathrm{~F} / \mathrm{dDP}$ from transactivating reporter constructs in cultured cells (Du et al., 1996), and in vivo overexpression of $\mathrm{RBF}$ in wing and eye imaginal cells inhibits cell cycle progression (Du et al., 1996; Neufeld et al., 1998). Loss of RBF function in embryos causes transcriptional derepression of $\mathrm{dE} 2 \mathrm{~F} / \mathrm{dDP}$ target genes and escape from $\mathrm{G}_{1}$ arrest (Du and Dyson, 1999). In addition, the $d M C M 3$ gene is derepressed in the CNS of $d D P$ mutant embryos (Duronio et al., 1998).

In spite of well-documented effects on cell cycle control, the entirety of the in vivo roles for E2F during animal development is not completely known. In particular, recent evidence suggests that certain mammalian E2F transcription factors contribute to the differentiation of specific cell types. Mouse E2F-5 is required only in one epithelial cell type in the brain, and does not have an essential role in cell cycle control (Lindeman et al., 1998). E2F-4 is abundant in postmitotic PC12 cells that have been induced to differentiate by exposure to nerve growth factor (NGF). Forced expression of E2F-4 prior to NGF addition enhances the ability of NGF to induce neurite outgrowth in these cells, and prolongs the differentiated state after NGF removal (Persengiev et al., 1999). These data suggest that E2F-4 contributes in some way to induction and/or maintenance of the neuronal cell fate. Similarly, Drosophila $d E 2 F$ is highly expressed in terminally differentiated, postmitotic cells of the retina, and seems to function in these cells as a survival factor and/or to maintain an appropriate differentiated phenotype (Brook et al., 1996).

In this report we have focused on DP function by extending our initial analysis of the $d D P$ mutant phenotype. The examination of zygotic $d D P$ mutant phenotypes is complicated by the perdurance of maternally supplied $\mathrm{dDP}$ protein, especially during embryonic development (Duronio et al., 1998). Thus, even at embryonic stages when obvious molecular phenotypes can be observed, the mutants are probably not null for dDP function. Certain biological roles for dDP may go undiscovered as a result. In order to overcome this problem and to search for additional functions for dDP, we generated $d D P$ mutant clones in the female germline to eliminate maternal stores of dDP. We anticipated that we would be able to generate mitotic clones of $d D P$ mutant cells since mutation of $d D P$ does not cause cell cycle arrest (Duronio et al., 1998; Royzman et al., 1997), and homozygous $d D P$ null mutant progeny progress to a very late stage of development (Royzman et al., 1997). Our results indicate that $\mathrm{dDP}$ is required in the germline for multiple processes during oocyte development. Most surprisingly, we observed a defect in the establishment of dorsal-ventral polarity in $d D P$ mutant egg chambers, indicating a novel developmental role for E2F transcription factors.

\section{MATERIALS AND METHODS}

\section{Genetics and fly stocks}

The Bloomington Drosophila Stock Center at Indiana University provided many of the strains used in these experiments. The $d D P^{a 2}$ EMS-induced mutation was provided to us by Terry Orr-Weaver (Royzman et al., 1997). The $d D P^{v r 10}$ EMS-induced mutation was originally designated vr10-13 (Duronio et al., 1998; Lasko and Pardue, 1988). Stocks of the genotype $\mathrm{P}\{\mathrm{hsFLP}\}^{1}, \mathrm{w}^{1118}$; $\mathrm{P}\left\{>\mathrm{w}^{\mathrm{hs}}>\right\} \mathrm{G} 13, d D P^{a 2} / \mathrm{CyO}$ and $\mathrm{P}\{\text { hsFLP }\}^{1}, \mathrm{w}^{1118} ; \mathrm{P}\left\{>\mathrm{w}^{\mathrm{hs}}>\right\} \mathrm{G} 13$, $\mathrm{dDP}^{\mathrm{vr} 10} / \mathrm{CyO}$ were generated using standard genetic techniques. The G13 P element is located at polytene position 42B and contains the FRT sites (designated as ' $>$ ') used for FLP induced recombination. The genotype of the ovo ${ }^{\mathrm{D}}$ stock used is $\mathrm{P}\left\{>\mathrm{w}^{\mathrm{hs}}>\right\} \mathrm{G} 13$ $\mathrm{P}\left\{\right.$ ovo $\left.^{\mathrm{D} 1-18}\right\} 32 \times 9$ a $\quad \mathrm{P}\left\{\right.$ ovo $\left.^{\mathrm{D} 1-18}\right\} 32 \times 9 \mathrm{~b} / \mathrm{Dp}(? ; 2) \mathrm{bw}^{\mathrm{D}}, \quad \mathrm{S}^{1} \quad \mathrm{wg}^{\mathrm{Sp}-1}$ $\mathrm{Ms}(2) \mathrm{M}^{1} \mathrm{bw}^{\mathrm{D}} / \mathrm{CyO}$.

\section{Construction of the dDP transgene}

The $d D P$ gene maps to polytene region 49F (Duronio et al., 1998). Cosmid clones containing genomic DNA from this region were obtained from the European Drosophila Genome Project (Institute of Molecular Biology and Biotechnology). Cosmid 130E2 was shown to contain $d D P$ by PCR analysis. A $2.2 \mathrm{~kb}$ BamHI-EcoRI fragment and a $4.3 \mathrm{~kb} E c o$ RI-EcoRI fragment from the $d D P$ gene were cloned separately into pBluescript (Fig. 1A,B). A $6.5 \mathrm{~kb} \mathrm{BamHI-XhoI}$ fragment from this clone, which contains all of the $d D P$ gene except for most of the large first intron, was subcloned into pCaSpeR 4. This construct was introduced into flies via $\mathrm{P}$ element mediated germline transformation. TM6 balancer chromosomes containing a $d D P \mathrm{P}$ element were isolated genetically by mobilizing the original transformant with exogenously provided transposase.

\section{Generation of germline clones}

To induce mitotic recombination, hsFLP/hsFLP; FRT dDP/CyO virgin females (genotype is an abbreviated version of that described above) were crossed to FRT, ovo $o^{D 1} / \mathrm{CyO}$ males and the resulting progeny were heat-shocked at $37^{\circ} \mathrm{C}$ for $3-4$ hours as late third instar larvae (72-96 hours old). FRTdDP/ovo ${ }^{D 1}$ mosaic progeny females were crossed with sibling or Canton-S males, and prepared for ovary dissection (see below) or egg collection. Eggs were counted on grape juice agar plates, or mounted in Hoyer's medium for photomicroscopy. The rescue experiment was performed in the same way, except that FRT, ovo ${ }^{D 1} /+$; TM6 $\mathrm{P}\left\{\mathrm{w}^{+} \mathrm{dDP}\right\} /+$ males were used. Since TM6 contains a dominant visible marker, we were able to distinguish mosaic FRT dDP/FRT ovo ${ }^{\mathrm{D} 1}$; TM6 $\mathrm{P}\left\{\mathrm{w}^{+} \mathrm{dDP}\right\} /+$ females from their FRT dDP/FRT ovo ${ }^{\mathrm{D} 1} ;+/+$ sisters. The rescue data for each $d D P$ allele shown in Tables 1 and 2 was generated using sibling flies, and represents a compilation of data obtained with two independent TM6 P $\left\{\mathrm{w}^{+} \mathrm{dDP}\right\}$ chromosomes.

There has been at least one report that additional germ cell mitoses are caused by nonspecific effects using the FLP/FRT ovo ${ }^{D 1}$ method of inducing mitotic recombination in the female germ line (Jordan and Karess, 1997). However, the penetrance of this 'background' phenotype was much lower $(<5 \%)$ than we observed in the $d D P$ mutant clones, and the recombination events were induced on a different chromosome ( $\mathrm{X}$ versus $2 \mathrm{R}$ ). Moreover, the $d D P$ phenotype was substantially rescued by a wild-type $d D P$ transgene (see Results).

\section{In situ hybridization analysis on ovaries}

Dissected ovaries were fixed in $200 \mu \mathrm{l} 5 \%$ formaldehyde in devitellinizing buffer $\left(16.7 \mathrm{mM} \mathrm{KH} 2 \mathrm{PO}_{4} / \mathrm{K}_{2} \mathrm{HPO}_{4}, \mathrm{pH} 6.9,75 \mathrm{mM}\right.$ $\mathrm{KCl}, 25 \mathrm{mM} \mathrm{NaCl}, 3.3 \mathrm{mM} \mathrm{MgCl}_{2}$ ) (Cant et al., 1994) and $600 \mu \mathrm{l}$ of heptane with gentle rocking for 10-20 minutes at room temperature. They were then washed $3 \times 2$ minutes in PBT $(1 \times$ PBS, $0.3 \%$ Triton $\mathrm{X}-100,0.5 \% \mathrm{BSA}$ ) and probed or stored in $100 \%$ ethanol at $-20^{\circ} \mathrm{C}$. Digoxigenin-labeled RNA probes from $R N R 2$ genomic DNA (Duronio and O'Farrell, 1994), dDP cDNA (Dynlacht et al., 1994), gurken cDNA (Neuman-Silberberg and Schüpbach, 1993) and oskar cDNA (Ephrussi et al., 1991) were generated. gurken and oskar cDNA were kindly provided by Trudi Schüpbach. Ovary in situ hybridization was performed on embryos as previously described for embryos 
Fig. 1. dDP mutant alleles used to analyze oogenesis. (A) The exon/intron structure of the $d D P$ gene is shown below a partial restriction map of a $10 \mathrm{~kb}$ piece of genomic DNA represented by the top line. $d D P$ is divergently transcribed from a gene of unknown function represented by two EST clones. We have not determined the extent of this transcript (dotted line). (B) For the phenotypic rescue experiments a $d D P$ 'mini' gene was constructed by ligating the BamHI$E c o$ RI and EcoRI-EcoRI fragments indicated by the two solid lines. (C) Diagram of the coding potential of the $d D P$ gene and the two $d D P$ alleles used in this study. (D) Diagram of a Drosophila ovariole (modified from Gilbert, 1994, with permission). In the germarium (bracket) germline stem cell daughters called cystoblasts divide 4 times to generate a 16-cell cyst, which becomes enclosed by a single layer of somatic follicle cells. One of these cells develops into the oocyte, while the other 15 become polyploid nurse cells.
A

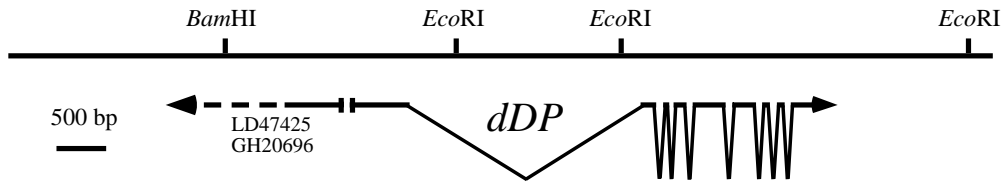

B

Rescue

Construct

C

DNA

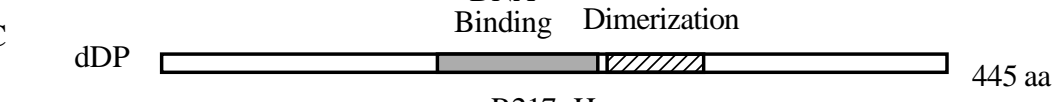

$\mathrm{R} 217>\mathrm{H}$

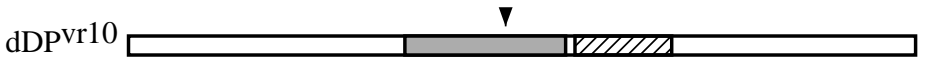

$\mathrm{D}$
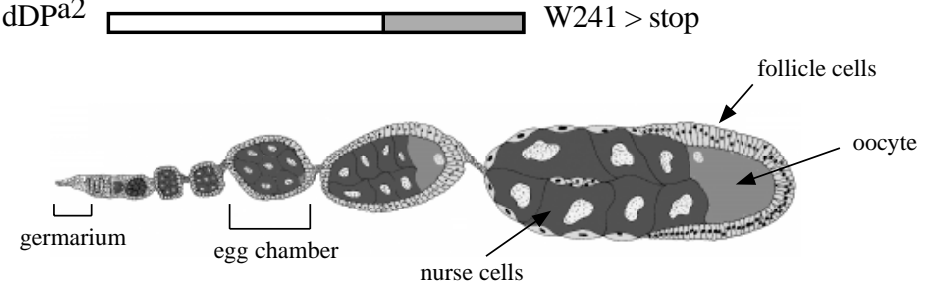

(Duronio and O'Farrell, 1994; Tautz and Pfeifle, 1989) with minor alterations. Prior to prehybridization ovaries were treated with 50 $\mu \mathrm{g} / \mathrm{ml}$ proteinase $\mathrm{K}$ for 5 minutes (Ephrussi et al., 1991), then washed for 5 minutes in $2 \mathrm{mg} / \mathrm{ml}$ glycine in PBT, followed by $3 \times 5$ minute PBT washes. The ovaries were postfixed in 5\% formaldehyde in PBT for 15 minutes followed by $5 \times 5$ minute washes in PBT. Hybridization occurred overnight at $55^{\circ} \mathrm{C}$. Alkaline phosphatase buffer washes contained $250 \mu \mathrm{g} / \mathrm{ml}$ levamisole. BM purple (Boehringer Mannheim) was used for detection. Ovaries were mounted in Fluoromount-G (Southern Biotechnology Associates, Inc.) for photographs on a Nikon Eclipse E800.

\section{Antibody staining of ovaries}

For Gurken antibody staining, ovaries were fixed in $200 \mu \mathrm{l} 4 \%$ paraformaldehyde in PMES (0.1 M Pipes, $\mathrm{pH} 6.9,2 \mathrm{mM} \mathrm{MgSO}_{4}, 1$ mM EGTA) containing $0.5 \%$ Nonidet P-40 and $600 \mu \mathrm{l}$ heptane with gentle rocking for 20 minutes at room temperature. Ovaries were rinsed in wash buffer $(50 \mathrm{mM}$ Tris, $\mathrm{pH} 7.4,150 \mathrm{mM} \mathrm{NaCl}, 0.5 \% \mathrm{NP}$ $40,1 \mathrm{mg} / \mathrm{ml} \mathrm{BSA}$ ), and then blocked in blocking buffer (50 mM Tris, $\mathrm{pH} 7.4,150 \mathrm{mM} \mathrm{NaCl}, 0.5 \% \mathrm{NP}-40,5 \mathrm{mg} / \mathrm{ml} \mathrm{BSA}$ ) for 2 hours at room temperature. They were then incubated overnight at $4^{\circ} \mathrm{C}$ with 1:3000 preabsorbed (with fixed ovaries from the grkHK36 mutant) rat anti-Gurken antibody (Neuman-Silberberg and Schüpbach, 1996) provided by Trudi Schüpbach. They were washed $4 \times$ for 20 minutes in antibody wash, incubated for 2 hours with 1:500 Cy3-conjugated goat anti-rat (Jackson Labs) secondary antibodies, and washed again $4 \times 20$ minutes in antibody wash, all at room temperature. Encore antibody staining was performed as for Gurken, using a 1:1000 dilution of the antibody provided by Trudi Schüpbach. Nuclei were stained using $1 \mu \mathrm{g} / \mathrm{ml}$ DAPI in PBT for 5 minutes. Filamentous actin was stained by incubating ovaries with gentle rocking for 20 minutes at room temperature in 1:1000 Alexa 488-conjugated phalloidin (Molecular Probes, Inc.). Ovaries were then washed $3 \times 5$ minutes in PBT. All ovaries were mounted in Fluoromount-G (Southern Biotechnology Associates, Inc.) and visualized using a Zeiss confocal microscope.

\section{TUNEL labeling of ovaries}

Dissected ovaries were fixed as for in situ hybridization analysis but were never placed in ethanol. Instead, after fixation they were retained in PBT and then rinsed once in PBS wash (PBS $+1 \%$ BSA). They were then rinsed in reaction buffer $\left(2.5 \mathrm{mM} \mathrm{CoCl}_{2}+1 \times\right.$ transferase buffer). After removal of this buffer, reaction mix $(1 \times$ transferase buffer $+\mathrm{CoCl}_{2}, 50$ units terminal transferase, 2:1 $10 \mu \mathrm{M}$ dUTP: biotin-16-dUTP) was added to the ovaries and they were incubated at $37^{\circ} \mathrm{C}$ for 3 hours. The terminal transferase kit was from Boehringer Mannheim. Processing was performed using the Vectastain kit (Vector Laboratories, Inc.) and fluorescence amplification using tyramide-Cy3 (1:100) (NEN Life Science Products, Inc.). Images were taken using a Zeiss confocal microscrope.

\section{RESULTS}

\section{dDP is required in the female germline for dorsal- ventral patterning}

To assess the role of maternal dDP in Drosophila we utilized two of the existing six $d D P$ alleles for germline clonal analysis (Fig. 1C). $d D P^{a 2}$ is a genetic null that contains a T-to-A nucleotide transition converting Trp173 to a stop codon (Royzman et al., 1997). Any truncated protein produced from this allele is likely to be nonfunctional, as part of the DNA binding domain and the entire region required for dimerization with dE2F would be missing. $d D P^{v r 10}$ is a semi-viable hypomorphic allele containing a missense mutation in the DNA binding domain that converts Arg217 to His (Duronio et al., 1998). This highly conserved residue makes a specific base contact in the E2F/DP DNA recognition site (Zheng et al., 1999), and thus $\mathrm{dDP} \mathrm{vr}^{\mathrm{vr} 10}$ mutant protein is expected to bind DNA poorly. The clonal analysis was performed using the FLP/FRT-dominant female sterile (DFS) technique in which homozygous $d D P$ mutant cells are produced in the female germline by FLPinduced mitotic recombination at FRT sites (Chou et al., 1993; Chou and Perrimon, 1996). The DFS mutation ovo ${ }^{D 1}$ provides a positive selection for the detection of germline mitotic recombination events, since germ cells that have not eliminated the $o v o^{D 1}$ mutation do not lead to the formation of eggs. 
Females carrying either $d D P^{a 2}$ or $d D P^{v r 10}$ germline clones were sterile. Mosaic $d D P^{a} /$ ovo ${ }^{D 1}$ females could produce some $d D P^{a 2}$ mutant eggs, but at a rate far less than wild-type flies. These eggs were all abnormal and none of them ever developed. The most striking abnormality was a highly penetrant defect of the chorion (egg shell). Normally a pair of respiratory appendages is located on the dorsal anterior side of the egg (Fig. 2A), but in the mutant eggs these were replaced by a single fused appendage (Fig. 2B). There was variability in the length and shape of the appendage, as well as in the degree of fusion; some appendages were fused only at the base, or fused at the base and at the tips but not in the middle (not shown). The mutant eggs were also smaller than normal, with some only half the size of wild-type eggs (not shown). Like the $d D P^{a 2}$ mutant eggs, $d D P^{v r 10}$ eggs displayed a range of different chorion phenotypes (Fig. 2; Table 1). While $17 \%$ of the eggs laid by $d D P^{v r 1 \%} / o v o^{D 1}$ mosaic females had two somewhat normal respiratory appendages (Fig. 2C), $80 \%$ had a single, fused appendage located at the dorsal midline (Fig. 2E,F). A small number (3\%) of eggs had either misplaced appendages or lacked appendages all together (Fig. 2D). When we examined the eggs laid from $d D P^{v r 10} / o v o D 1$ mosaic females, $99 \%$ did not develop to the stage when a cuticle is produced; they were either never fertilized or did not develop sufficiently that a cuticle was secreted. Of the small number of embryos that did develop cuticles, several displayed severe but variable pattern defects while others appeared normal (not shown). Occasionally a larva would hatch from an egg laid by a $d D P^{\text {vrlo }} \% o^{D 1}$ mosaic female. These rare individuals were presumably rescued by $\mathrm{dDP}$ function provided zygotically from a wild-type paternal chromosome.

These data indicate that $\mathrm{dDP}$ function is required maternally, and are consistent with the observation that $d D P$ mRNA is expressed throughout oogenesis (Royzman et al., 1999; D. L. Myster, unpublished data). In particular, the defects in eggshell morphology indicated that $\mathrm{dDP}$ was required for proper patterning during oogenesis. Fusion of the respiratory appendages at the dorsal midline suggests a ventralized egg shell, indicative of a failure to properly establish dorsal fate in the follicular epithelium of the egg chamber. We therefore conducted a phenotypic analysis of ovaries from $d D P$ mosaic females.

\section{$d D P$ mutant egg chambers display a 'dumpless' phenotype}

A Drosophila ovary contains 12-14 ovarioles that each consist of a series of individual egg chambers at successive stages of development (Fig. 1D) (Spradling, 1993). Each egg chamber contains 16 germ cells derived from a single precursor cell that executes exactly four mitoses. These 16 sister cells remain connected through cytoplasmic bridges called ring canals, due to incomplete cytokinesis. This germ cell cyst is enclosed by an epithelium consisting of a single layer of somatic follicle cells. One of the 16 germ cells becomes the oocyte, and the remaining 15 develop as polyploid nurse cells that support oocyte growth. The nurse cells synthesize large amounts of RNA and protein that are transported to the oocyte through the ring canals.

Using combinations of $d D P$ alleles that permit development but cause female sterility, Royzman et al. (1999) demonstrated previously that dDP function is required in the ovary for the amplification of chorion loci in the follicle cells. As a result, the eggs from these $d D P$ mutant mothers have thin egg shells and do not develop. In our experiments clones of $d D P$ mutant cells are generated throughout the animal, possibly including the follicle cells; however, the phenotypes we describe below are due to a dDP requirement in the germline rather than the follicle cells. First, the penetrance of the fused respiratory appendage phenotype is very high (>75\% for $d D P^{v r 10}$ and usually $90-100 \%$ for $d D P^{a 2}$ ). The chance that this results from loss of dDP function specifically in the dorsal follicle cells that
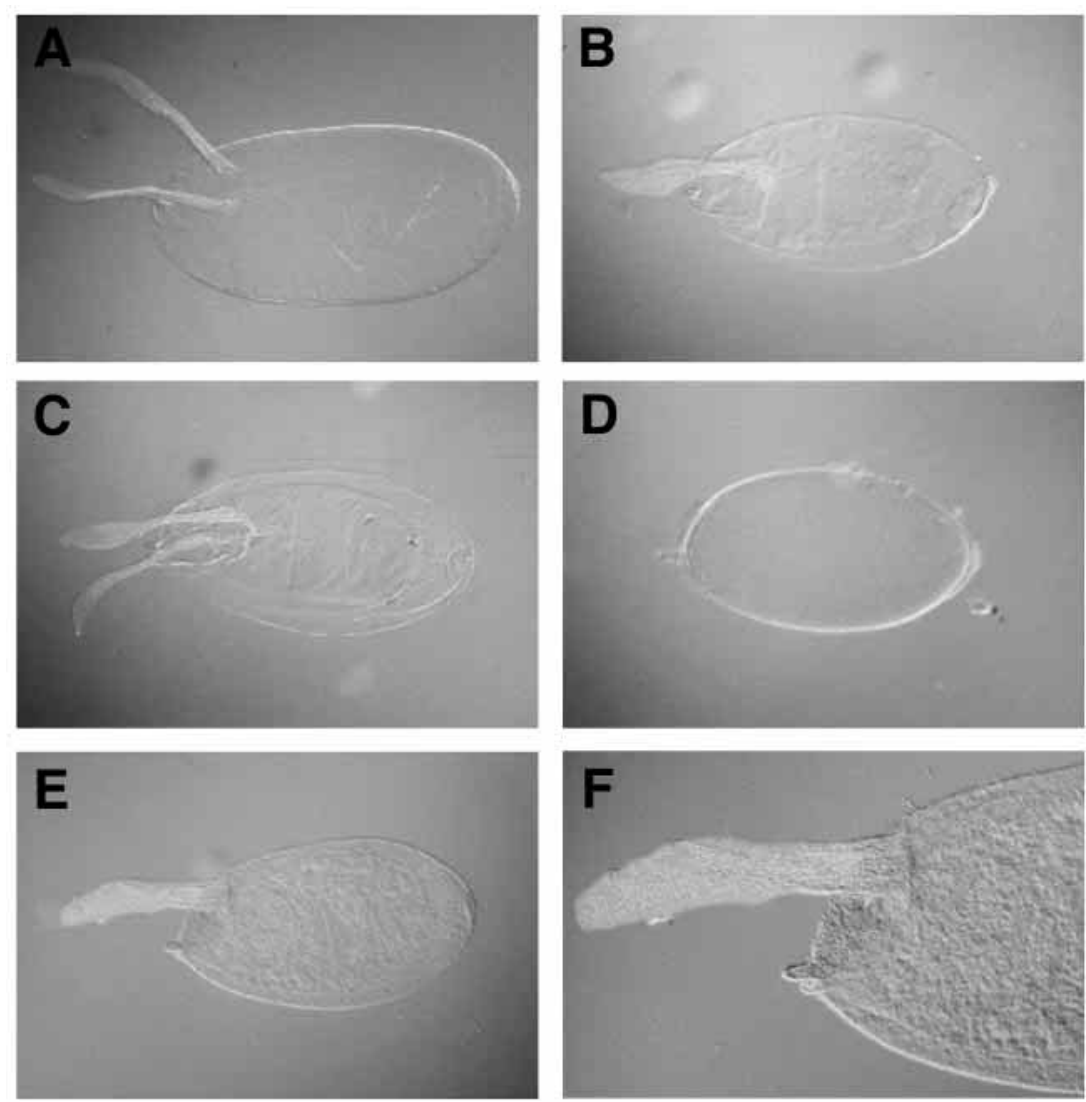

Fig. 2. dDP is required for proper dorsal-ventral patterning of the eggshell. Eggs laid by females carrying either $d D P^{a 2}(\mathrm{~B})$ or $d D P^{v r 10}(\mathrm{C}-\mathrm{F})$ germline clones displayed a range of different eggshell phenotypes. (A) Wild-type eggs contain a pair of respiratory appendages on the dorsal anterior side of the egg. (B) Almost all eggs laid by $d D P^{a 2} / o v o^{D 1}$ females had a single fused dorsal appendage. Appendages were often short and wide and eggs were smaller than wild-type. (C) 15-20\% of eggs laid by $d D P^{v r 10} \% v o^{D 1}$ females contained two dorsal appendages. Some of these appendages were not completely normal in shape and/or length and often were placed too close together or even fused at the base. (D) A small percentage of mutant eggs from both alleles contained no appendages at all. (E) $d D P^{v r 10} / o v o^{D 1}$ females also laid a high proportion of eggs with a single fused dorsal appendage. (F) Higher magnification shows a closer view of the $d D P^{v r 10}$ fused appendage. (A-E) $10 \times$; (F) $20 \times$ magnification. 
Table 1. Distribution of eggshell phenotypes in $d D P$ germline clones

\begin{tabular}{|c|c|c|c|c|c|}
\hline \multirow[b]{2}{*}{ Genotype } & \multicolumn{5}{|c|}{ Eggshell phenotypes } \\
\hline & $\begin{array}{c}\text { Two } \\
\text { appendages } \\
(\%)\end{array}$ & $\begin{array}{c}\text { Fused } \\
\text { appendages } \\
(\%)\end{array}$ & $\begin{array}{c}\text { No } \\
\text { appendages } \\
(\%)\end{array}$ & $\begin{array}{c}\text { Number } \\
\text { scored }\end{array}$ & $\begin{array}{c}\text { Hatching } \\
(\%)\end{array}$ \\
\hline Canton-S & 100 & 0 & 0 & 658 & ND \\
\hline $\mathrm{dDP}^{\mathrm{vr} 10} / \mathrm{ovo}^{\mathrm{D} 1}$ & 17 & 80 & 3 & 7,029 & 0.7 \\
\hline 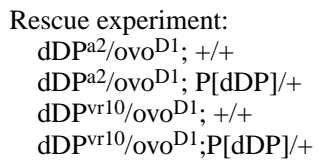 & $\begin{array}{l}14 \\
96 \\
22 \\
95\end{array}$ & $\begin{array}{r}78 \\
2 \\
75 \\
3\end{array}$ & $\begin{array}{l}8 \\
2 \\
3 \\
2\end{array}$ & $\begin{array}{r}95 \\
503 \\
624 \\
2,061\end{array}$ & $\begin{array}{r}0 \\
43 \\
0 \\
26\end{array}$ \\
\hline
\end{tabular}

See text for details.

ND, not determined.

synthesize the appendages is small. Second, all the phenotypes we describe were observed in egg chambers that have developed past the $o v o^{D 1}$ arrest point, which can only occur from a mitotic recombination event in the germline.

Ovarioles dissected from mosaic $d D P^{a 2} /$ ovo ${ }^{D 1}$ females contained $d D P^{a 2}$ homozygous mutant egg chambers that progressed to the final stages of oogenesis, and deposited chorion material around the oocyte. As with the $d D P^{a 2}$ eggs, these late-stage oocytes were much smaller than normal and the chorions were ventralized (not shown). Near the end of oogenesis the nurse cells rapidly transport all of their cytoplasmic contents through the ring canals into the oocyte and apoptose (Foley and Cooley, 1998; McCall and Steller, 1998; Robinson and Cooley, 1997b). Mutants that are defective in this process display a 'dumpless' phenotype that results in small oocytes like those seen in the $d D P^{a 2}$ mutant egg chambers. We detected a failure of nurse cell cytoplasmic transfer using in situ hybridization with a probe derived from the RNR2 gene, which is normally transcribed in nurse cells and then loaded into the oocyte (Fig. 3A). In $100 \%$ of the late-stage $d D P^{a}$ mutant egg chambers the nurse cells retained high amounts of $R N R 2$ message (Fig. 3B) while counterpart wild-type nurse cells no longer did. The dumpless phenotype was also apparent in some of the $d D P^{v r 10}$ mutant egg chambers (Fig. 3C), and in ovaries removed from sterile females carrying the hypomorphic allele combination $d D P^{a l} /$ Deficiency (T. Orr-Weaver, personal communication). In some egg chambers a double rather than single layer of follicle cells appeared in certain areas around the oocyte (e.g. see Fig. 7E), perhaps because the wild-type number of follicle cells exceeds that needed to encompass the small oocyte. We conclude from these data that $\mathrm{dDP}$ is required for nurse cell cytoplasmic transfer.

Disruption of the actin cytoskeleton or the integrity of the actin-rich ring canals blocks cytoplasmic transport between the nurse cells and the oocyte (Cant et al., 1994; Cooley et al., 1992; Mahajan-Miklos and Cooley, 1994; Robinson et al., 1997; Xue and Cooley, 1993). Filamentous actin can be readily visualized in Drosophila egg chambers with fluorophore-conjugated phalloidin. Prior to the dumping stage in both wild-type and $d D P$ mutant egg chambers, most of the actin accumulates at the cortex of the follicle cells, nurse cells and oocyte, and in the ring canals (Figs 4A,B, 6D-F). However, in both $d D P^{a 2}$ and $d D P^{v r 10}$ mutant oocytes we detected aberrant accumulation of actin in several brightly stained foci, which appeared in young egg chambers (Fig. 4B, arrow) and persisted throughout oogenesis (Fig. 6E, arrow). Although
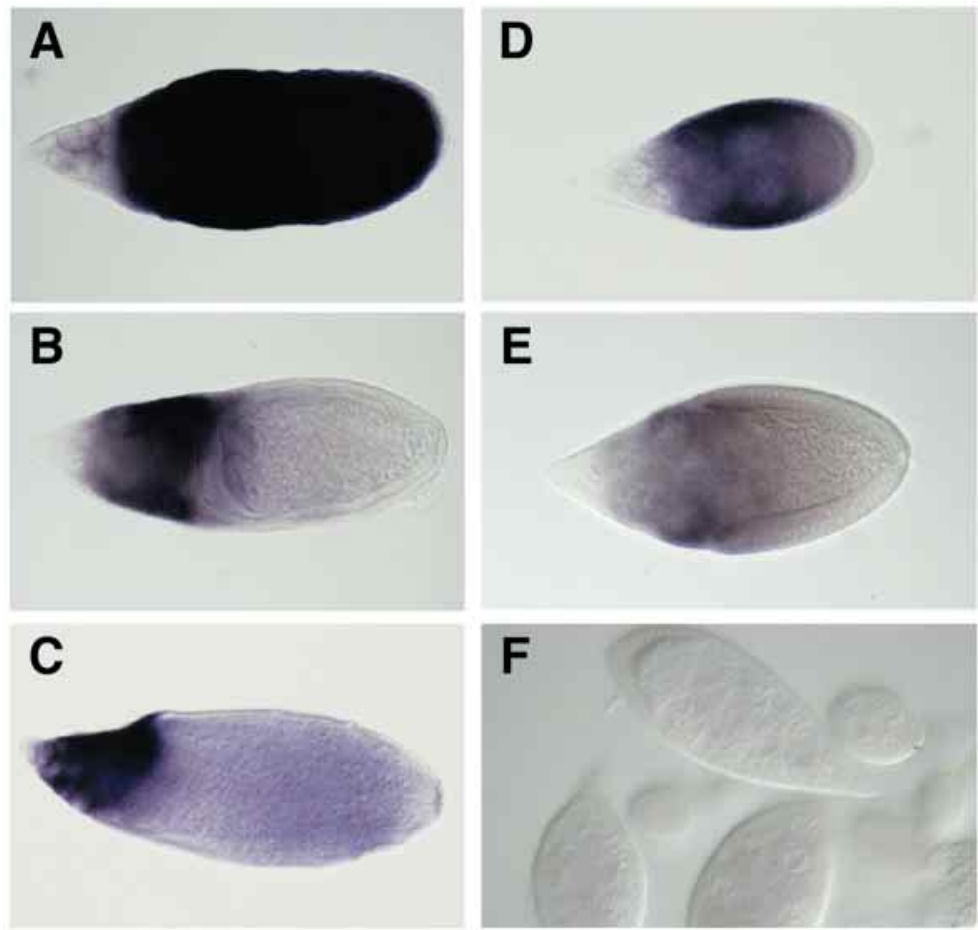

Fig. 3. $d D P$ mutant egg chambers display a 'dumpless' phenotype. Ovaries dissected from mosaic $d D P^{a 2} / o v o^{D 1}$ or $d D P^{v r 10} / o v o^{D 1}$ females were analyzed by in situ hybridization for $R N R 2$. (A) $R N R 2$ mRNA is normally transcribed in the nurse cells and then loaded into the oocyte by stage 11 as seen in this egg chamber. Nurse cells of late-stage $d D P^{a 2}$ (B) or $d D P^{v r 10}$ (C) mutant egg chambers retained high amounts of $R N R 2$ message. Younger $d D P$ mutant egg chambers (stages 8-9) display a reduced level of $R N R 2$ mRNA expression. (D) In situ hybridization for $R N R 2$ showed a high level of the message in the oocyte and nurse cells throughout oogenesis prior to nurse cell transport, as in this stage- 8 oocyte. (E) There was a moderate but consistent reduction of $R N R 2$ expression in $d D P^{a 2}$ mutant egg chambers compared to wild type, as seen in this stage- 9 oocyte. This reduction was not as evident in $d D P^{v r 10}$ mutant egg chambers. (F) A sense in situ probe for $R N R 2$ revealed little to no background staining of ovaries and also confirmed that the staining in $d D P$ mutant chambers is above background. 

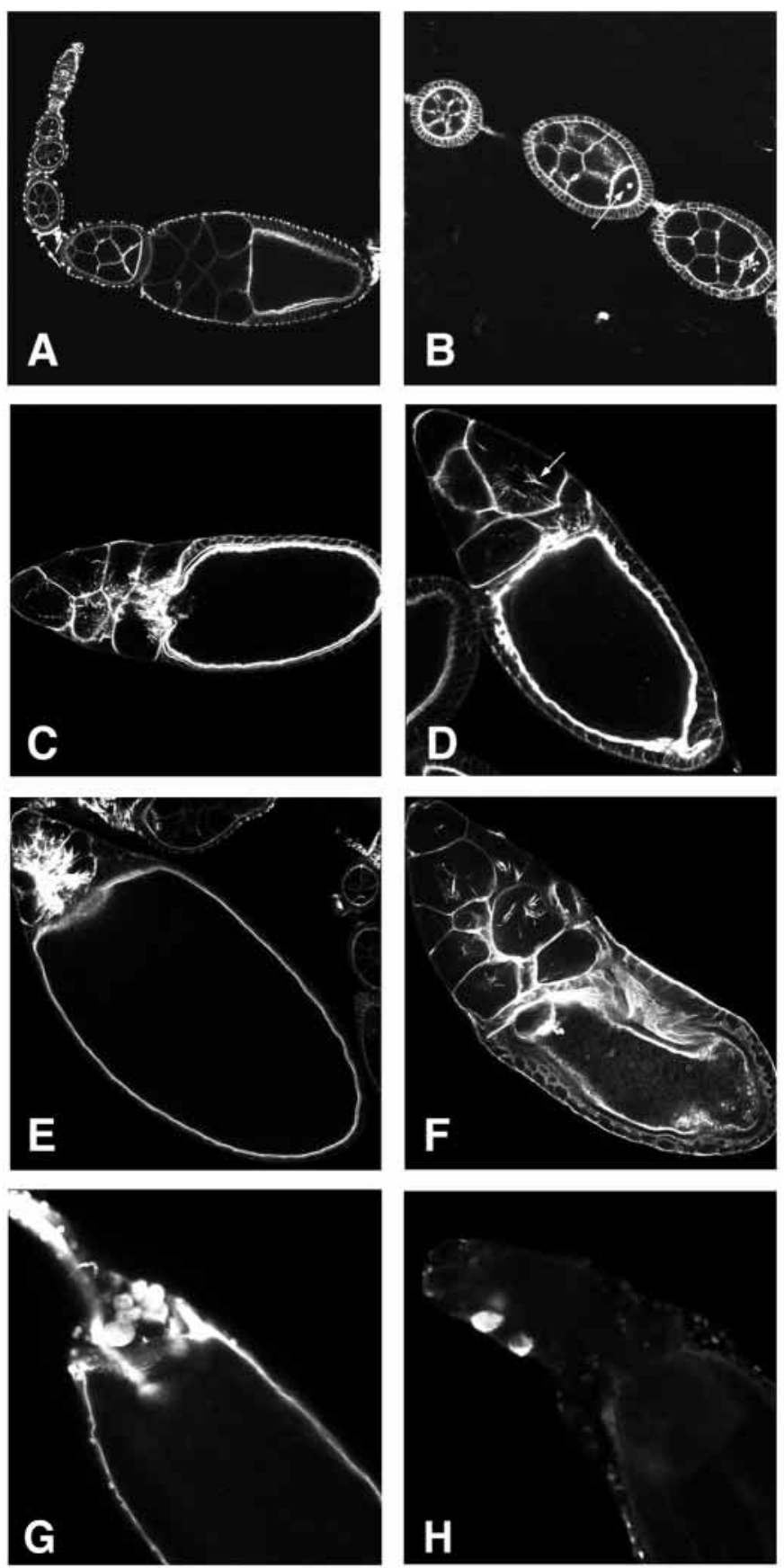

their origin is unclear, these foci indicate that the actin cytoskeleton is perturbed by loss of dDP function. A defect was also apparent in late-stage $d D P$ mutant egg chambers. Normally in wild type, dense bundles of actin filaments form in the nurse cell cytoplasm during the dumping stage (Fig. $4 \mathrm{C}, \mathrm{E})$. These bundles are required for nurse cell to oocyte cytoplasmic transfer (Mahajan-Miklos and Cooley, 1994). Actin bundles did form in the $d D P^{a 2}$ and $d D P^{v r 10}$ mutant nurse cells, but their density was reduced compared to wild type (Fig. 4D,F).

$d D P^{a 2}$ mutant nurse cells failed to degenerate on schedule, as revealed by DAPI (not shown) and phalloidin staining (Fig. $4 F$ ). This suggested that dDP may play a role in nurse cell apoptosis, similar to recently published data showing that
Fig. 4. Actin organization in $d D P$ mutant egg chambers. Filamentous actin was visualized in egg chambers using fluorophore-conjugated phalloidin. (A) In young egg chambers, prior to nurse cell dumping, actin accumulates at the cortex of follicle cells, nurse cells and the oocyte, as well as in ring canals. (B) This early accumulation of actin is normal in $d D P$ mutant egg chambers. However, aberrant actin accumulates in bright foci (arrow) in the oocytes of these young egg chambers. (C) Wild-type nurse cell nuclei degenerate, starting at stage 12 during oogenesis. Coincident with nurse cell dumping and nuclei degeneration are actin cytoskeleton rearrangements in the nurse cells. Cytoplasmic actin bundles form by stage 10B and are seen by confocal microscopy in this wild-type egg chamber. (D) Stage-10B $d D P$ mutant egg chambers have some cytoplasmic actin bundles (arrow) although they appeared at reduced levels compared to wild type. (E) Dense actin bundles remain in the nurse cells of this wild-type stage-12 egg chamber that has gone through nurse cell dumping. Cortical actin staining is present in the follicle cells of these egg chambers but is hard to visualize in this chamber. (F) $d D P^{a 2}$ or $d D P^{v r 10}$ mutant nurse cells fail to degenerate on time, as revealed by actin staining in this late-stage mutant egg chamber. Actin bundles are present but again at a reduced level. (G) TUNELlabeling reveals nurse cell apoptosis in a stage-13 wild-type egg chamber. (H) A similar stage $d D P^{a}$ dumpless mutant egg chamber contains only two TUNEL-positive nurse cell nuclei.

mutation of the DCP-1 caspase prevents nurse cell apoptosis and causes a dumpless phenotype (McCall and Steller, 1998). Interestingly, $d c p-1$ mutants also fail to form cytoplasmic actin bundles (McCall and Steller, 1998). We therefore decided to test whether $d D P^{a 2}$ mutant egg chambers were defective in apoptosis. TUNEL labeling was used to detect the $3^{\prime}$ ends of fragmented DNA in apoptotic cells in ovaries. Wild-type nurse cell nuclei were labeled during stages 12 and 13 of oogenesis (Fig. 4G), as seen in previous studies (Foley and Cooley, 1998; McCall and Steller, 1998). However, we discovered that $d D P^{a 2}$ dumpless stage-13 egg chambers contained few if any nurse cells that were TUNEL positive (Fig. 4H). Stage-13 egg chambers that contained a single fused dorsal appendage had at most 2-3 labeled nuclei. Participation of dDP in nurse cell apoptosis provides a possible explanation for the dumpless phenotype of the $d D P$ mutant clones, and is consistent with a known requirement for $E 2 F-1$ for apoptosis in certain cell types in the mouse (Field et al., 1996; Pan et al., 1998; Tsai et al., 1998).

The respiratory appendages of the eggshell are secreted by specialized follicle cells, which migrate over the dorsal anterior portion of the oocyte as the nurse cells are degenerating (Spradling, 1993). The failure of nurse cells to transfer their contents to the oocyte can apparently affect the ability of these follicle cells to properly form the respiratory appendages. kelch encodes a component of the actin-rich ring canals, and is required for nurse cell dumping (Robinson and Cooley, 1997a; Xue and Cooley, 1993). Fused respiratory appendages have been observed in some kelch mutant egg chambers (Gutzeit and Straub, 1989; Xue and Cooley, 1993), possibly because follicle cell migration is impeded by nurse cells that have not degenerated (Schüpbach and Wieschaus, 1991). However, the dumpless phenotype does not always lead to fused respiratory appendages along the dorsal midline (Rittenhouse and Berg, 1995). Moreover, the phenotype of the $d D P^{v r 10}$ mutant eggs suggests that a dumpless phenotype is not the sole cause of the defect in appendage formation. Although we could detect a 
dumpless phenotype in $d D P^{v r 10}$ egg chambers (Fig. 3C), it appeared to be less severe than in $d D P^{a 2}$, and many of the eggs laid by the $d D P^{v r 10}$ mothers were of normal size. Importantly, a single, fused dorsal appendage was often observed in $d D P^{v r 10}$ mutant eggs that were wild type in size (not shown) (Manseau et al., 1996). The single appendage was often of typical length, suggesting that migration of the follicle cells proceeded normally. Thus, we interpret the fused respiratory appendage along the dorsal midline in the $d D P^{a 2}$ and $d D P^{v r 10}$ eggs as an indication that the eggshell is ventralized through a disruption of the normal process that determines dorsal follicle cell fate.

\section{dDP mutant oocytes have defective Grk expression}

Dorsal-ventral patterning of the egg and developing embryo begins with an asymmetry during oogenesis that becomes most apparent in the formation of the eggshell. Dorsal-ventral polarity requires communication between the developing oocyte and the overlying somatic follicle cell layer (Schüpbach, 1987; reviewed in Ray and Schupbach, 1996). The Drosophila Gurken(Grk) protein, a member of the TGF $\alpha$ family of growth factors, is expressed in the oocyte and activates the receptor Torpedo/Egfr (Epidermal growth factor receptor) found in the follicle cell epithelium. This Grk-Egfr signaling pathway is required early in oogenesis to establish anterior-posterior polarity and later in oogenesis to establish dorsal-ventral polarity (Gonzalez-Reyes et al., 1995; NeumanSilberberg and Schupbach, 1993; Roth et al., 1995). Mutations of $g r k$ and female sterile alleles of Egfr cause ventralization of eggshells and embryos. In particular, hypomorphic alleles of grk result in a single fused respiratory appendage at the dorsal midline (Schüpbach, 1987).

Since germline clones of $d D P$ mutations cause ventralized eggshells, we wanted to determine if this was due to an effect on grk expression. The expression of grk mRNA in mutant ovaries was first examined by in situ hybridization. In early stage (up to stage 7) wild-type egg chambers grk mRNA is concentrated in the oocyte. While $d D P^{a}$ mutant egg chambers are indistinguishable from $o v o^{D 1}$ chambers at these early stages, we did not observe any abnormalities in grk expression at these stages; thus we assume that $\mathrm{dDP}$ is not required for early expression of $g r k$ in the oocyte. During stage 8 , grk mRNA is briefly found in an anterior ring in the oocyte, and by stage 10 becomes localized around the oocyte nucleus, which has migrated to the dorsal-anterior side of the oocyte (Fig. 5A) (Neuman-Silberberg and Schüpbach, 1993). Some oocytes of stage-8 and $-10 d D P^{a 2}$ mutant egg chambers contained localized grk mRNA similar to wild type, displaying anterior rings followed by an accumulation around the oocyte nucleus (Fig. 5B). Thus, dDP is not absolutely required for localized grk expression in the oocyte. We did, however, observe aberrant expression of grk in the nurse cells of other developing egg chambers by stage 8 . Wild-type stage-7 to stage-10b egg chambers contain very little expression of grk in nurse cells, but there was a reproducible increase in the amount of grk mRNA in the cytoplasm of nurse cells in some $d D P^{a 2}$ mutant egg chambers (Fig. 5C). Although the basis for this phenotype is unclear, it may reflect a disruption of the unidirectional cytoplasmic flow from nurse cell to oocyte, causing grk mRNA synthesized by the oocyte nucleus to accumulate in the nurse cells. Alternatively, dDP may be required to repress grk transcription in the nurse cells.
Since $g r k$ mRNA appeared to be localized properly in some oocytes, we went on to analyze whether Grk protein expression was affected in our germline mutants. Normal Grk protein expression is very similar to grk mRNA expression. The protein is detected in the germarium and then localized early to the oocyte. By stage 10, Grk is found in a dorsal-anterior patch over the oocyte nucleus (Fig. 6A) and then later forms a stripe posteriorly away from the nucleus, reflecting Grk accumulation in the oocyte plasma membrane (NeumanSilberberg and Schupbach, 1996). Using anti-Grk antibodies we discovered a consistent reduction in the amount of detectable Grk protein in $d D P^{2}$ mutant egg chambers (Fig. $6 C)$. $d D P^{v r 10}$ mutant egg chambers revealed an additional phenotype. Grk protein was often detected in localized foci around the oocyte nucleus (Fig. 6B). The Grk protein failed to accumulate in the normal pattern in the oocyte plasma membrane, suggesting that Grk protein was not properly transported or anchored in the $d D P^{v r 10}$ mutant oocytes. This mislocalized Grk protein was also associated with the aberrant actin accumulation in the oocyte (Fig. 6E). Thus, the defect in the actin cytoskeleton may prevent Grk translocation to the cell surface. Since Grk is required to signal dorsal follicle cell fate, mutants that inhibit Grk accumulation or localization block Grk signaling. Thus the ventralization of the eggshell caused by mutation of $d D P$ in the germline is likely to be due to reduction and/or mislocalization of Grk protein in the oocyte.

Prior to its role in signaling dorsal follicle cell fate, Grk is required to establish the anterior-posterior axis of the oocyte by signaling to the posterior follicle cells. oskar is a member of the posterior group genes and has been shown to organize the germ plasm and direct localization of germ plasm components such as nanos mRNA (Ephrussi et al., 1991). In wild-type egg chambers oskar mRNA is synthesized by nurse cells and then accumulates in the oocyte. By stage 9, oskar mRNA becomes localized to the posterior of the oocyte where it remains throughout oogenesis (Ephrussi et al., 1991; KimHa et al., 1991). This localization requires Grk signaling, which initiates anterior-posterior polarity in the oocyte. In situ hybridization of $d D P^{a 2}$ mutant egg chambers indicated that oskar mRNA was localized to the posterior end of the oocyte (not shown), suggesting that lack of dDP function does not affect this early aspect of Grk signaling.

\section{dDP functions in germline cell cycle control}

None of the phenotypes thus far described have any obvious connection to cell cycle regulation. There is apparently no overt defect in cell cycle progression in the $d D P$ mutant germ cells, as egg chambers were obtained with relatively high frequency. Using DNA binding dyes such as DAPI (Fig. 7A) or propidium iodide we observe polyploid nurse cell nuclei in $d D P$ mutant egg chambers (Fig. 7B), suggesting that these nuclei have executed the normal program of endoreduplication cycles. Nurse cell nuclear DNA was also shown to replicate, based on BrdU incorporation during oogenesis (not shown). We have not determined whether the extent of polyploidization in the mutant nurse cells was similar to wild type, nor have we determined whether dDP contributes in more subtle ways to chromosome endoreduplication, such as the prevention of heterochromatin rereplication, which occurs in cyclin $E$ hypomorphic mutants (Lilly and Spradling, 1996).

In contrast to the presumed role in stimulating cell cycle 
progression, we observed a defect in the cessation of germ cell division caused by mutation of $d D P$. The number of DAPIstained nurse cell nuclei was approximately double that of wild type in $26 \%$ and $10 \%$ of the $d D P^{a 2}$ and $d D P^{v r 10}$ mutant egg chambers, respectively (Table 2 and Fig. 7B). Two possible ways that such egg chambers could arise are (1) that follicle cells could incorrectly package two 16-cell cysts together producing a 32-cell egg chamber, or (2) exactly one extra round of mitosis could occur to generate 32 rather than 16 cells. The former case would result in an egg chamber that had two oocytes, each with four ring canals, while the latter would produce an egg chamber containing one oocyte with five ring canals instead of four. We attempted to distinguish between these possibilities by simultaneously staining $d D P^{a 2}$ mutant ovaries with phalloidin to detect ring canals and with DAPI to count nuclei. Individual egg chambers with extra nurse cells were subjected to optical sectioning by confocal microscopy. By this analysis we could unambiguously detect egg chambers containing a single oocyte with five ring canals (Fig. 7C-E). This suggests that in these particular egg chambers $(n=5)$ an additional germline mitosis has occurred (Hawkins et al., 1996). This data indicates that dDP plays a role in terminating cell division in the germline.

In situ hybridization for oocyte-specific messages such as grk, as well as phalloidin staining, revealed that some egg chambers with approximately double the number of nurse cell nuclei also contained two oocytes. Egg chambers from females homozygous for a female sterile allele of cyclin $E$ sometimes contain 2-3 oocytes, suggesting that defects in cell cycle control affect oocyte determination (Lilly and Spradling, 1996). Since cyclin $E$ is a transcriptional target of E2F/DP in Drosophila, the occurrence of two oocytes in $d D P$ mutant egg chambers may result from a reduction of cyclin $E$ function, which disrupts oocyte determination.

Since $d D P$ is required for the expression of genes encoding replication factors in the embryo, we wished to determine if $d D P$ had a similar function during oogenesis. In situ hybridization analysis indicates that $R N R 2$ is expressed throughout wild-type egg chambers for the duration of oogenesis. There was a moderate reduction in the expression of $R N R 2$ in stage- 8 to $-9 d D P^{a}$ mutant egg chambers compared to wild type (cf. Fig. 3D,E). At these stages the nurse cells are endoreduplicating. Thus, these data suggest

Fig. 6. $d D P$ mutant oocytes have altered Gurken expression. Ovaries dissected from mosaic $d D P^{a 2} / o v o^{D 1}$ or $d D P^{v r 1 \%} / o v o^{D 1}$ females were double stained for Gurken (Grk) protein (A-C) and filamentous actin (D-F). (A) Grk is localized to the dorsal-anterior side of the oocyte, around the oocyte nucleus, by stage 9 in wild-type egg chambers. (B) Immunostaining and confocal microscopy detected Grk protein mislocalized in foci around the oocyte nucleus of $d D P^{v r 10}$ mutant egg chambers (arrow). (C) $d D P^{a 2}$ mutant egg chambers displayed a consistent reduction in the amount of detectable Grk protein (arrow).

(D) Corresponding wild-type actin staining.
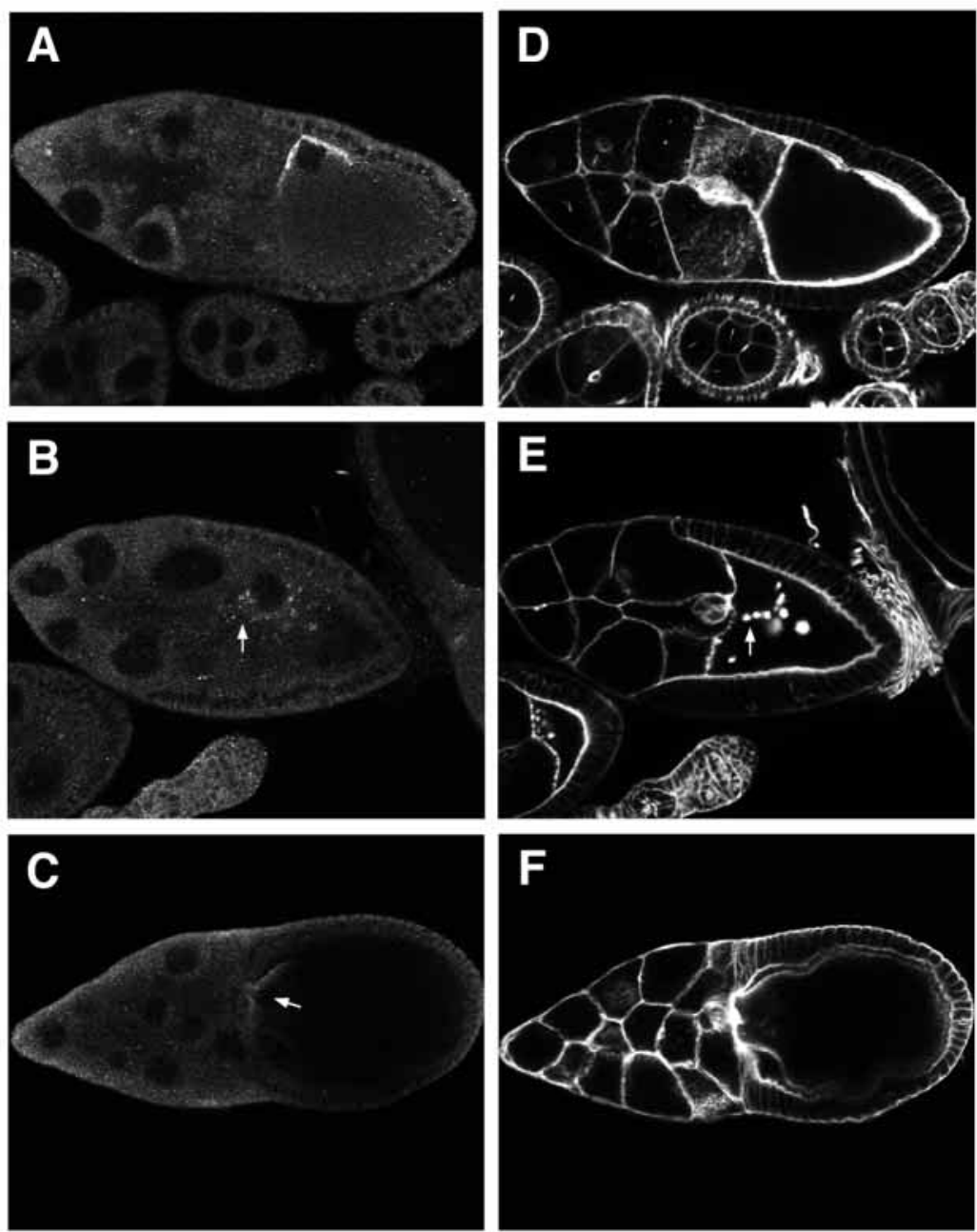

(E) Mislocalized Grk protein in $d D P^{v r 10}$ mutant egg chambers colocalizes with aberrant actin accumulation (arrow) seen in the oocytes of these mutant egg chambers. (F) Note that this $d D P^{a 2}$ egg chamber has an excessive number of nurse cells.
Fig. 5. Expression of grk mRNA in $d D P$ mutant egg hybridization shows dorsalanterior grk mRNA nucleus of this wild-typ
stage-10 egg chamber. (B) Some stage-10 dDP $P^{2}$ mutant egg chambers grk mRNA, as shown here. (C) Aberrant expression of gr some $d D P^{a 2}$ mutant egg chambers. cytoplasm of the nurse cells of
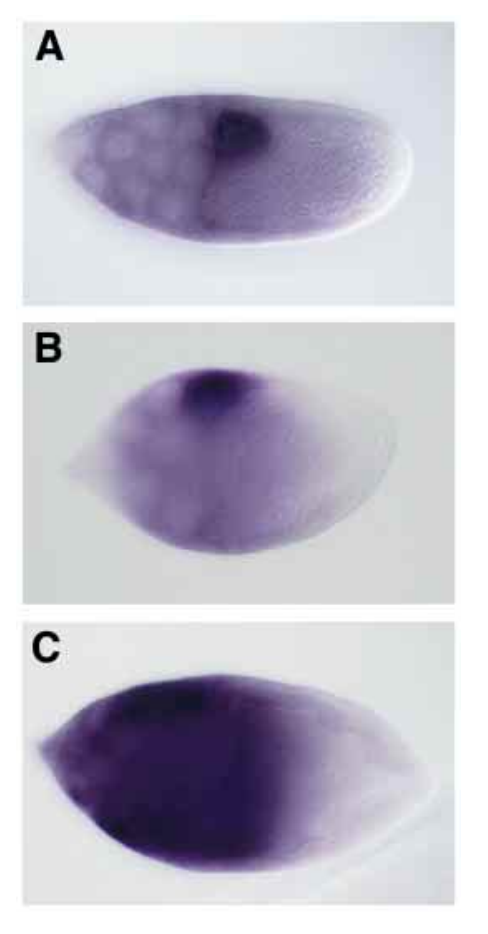
Table 2. Distribution of egg chambers with too many nurse cells in $d D P$ germline clones

\begin{tabular}{|c|c|c|c|}
\hline \multirow[b]{2}{*}{ Genotype } & \multicolumn{3}{|c|}{ Distribution of egg chambers } \\
\hline & $\begin{array}{c}\text { Egg chambers } \\
\text { with approx. } \\
15 \text { nurse cells } \\
(\%)\end{array}$ & $\begin{array}{c}\text { Egg chambers } \\
\text { with approx. } \\
30 \text { nurse cells } \\
(\%)\end{array}$ & $\begin{array}{c}\text { Number } \\
\text { scored }\end{array}$ \\
\hline Canton-S & 100 & 0 & 45 \\
\hline $\mathrm{dDP}^{\mathrm{a} 2} / \mathrm{ovo} \mathrm{D}^{\mathrm{D} 1}$ & 74 & 26 & 89 \\
\hline $\mathrm{dDP}^{\mathrm{vr} 10} / \mathrm{ovo}^{\mathrm{D} 1}$ & 90 & 10 & 82 \\
\hline 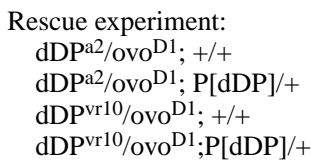 & $\begin{array}{l}88 \\
95 \\
93 \\
99\end{array}$ & $\begin{array}{r}12 \\
5 \\
7 \\
1\end{array}$ & $\begin{array}{r}34 \\
100 \\
59 \\
67\end{array}$ \\
\hline
\end{tabular}

See text for details

that $\mathrm{dDP}$ function contributes to $R N R 2$ expression during nurse cell endoreduplication cycles, just as in embryos. Although the difference was readily discernible and reproducible, there was not a complete loss of $R N R 2$. By stage $10 R N R 2$ mRNA was readily detectable in both wild-type and mutant egg chambers (not shown). At this stage the nurse cells have stopped replicating and have begun to synthesize large amounts of mRNA, which is destined for transport to the oocyte (Fig. 3A). Thus, dDP does not appear to be limiting during this stage of oogenesis.

\section{A wild-type dDP transgene complements the mutant phenotypes}

In order to confirm that the phenotypes we have described are due specifically to loss of $\mathrm{dDP}$ function, a molecular complementation experiment was performed. A transgene containing a 'mini' version of the wild-type $d D P$ gene lacking most of the large first intron was constructed in a $\mathrm{P}$ element vector (see Fig. 1B). This transgene is capable of rescuing the zygotic lethality of the $d D P$ null phenotype (T. Grana and R. J. Duronio, unpublished). Mitotic clones were generated as before in $d D P^{a 2} / o v o^{D 1}$ and $d D P^{v r 10} / o v o^{D 1}$ heterozygous flies carrying a copy of this transgene (see Materials and Methods). The majority of eggs laid by these mosaic females were wild type in size and produced a normal chorion with two respiratory appendages, while only $2-3 \%$ of the eggs contained a fused dorsal appendage and $2 \%$ had no appendages (Table 1 ). Moreover, many of these eggs hatched $(26 \%$ and $43 \%$ for

Fig. 7. dDP functions to stop germline cell division at the appropriate time. DAPI staining was used to observe nuclei in mutant egg chambers. (A) Wild-type egg chambers contain 15 nurse cell nuclei. (B) DAPI staining revealed that a percentage of $d D P^{a 2}$ and $d D P^{v r 10}$ mutant egg chambers contain approximately twice the normal number of nurse cell nuclei (approx. 30). One extra round of germline mitosis generates an oocyte with five ring canals instead of four. Phalloidin staining highlights oocyte ring canals. Some $d D P$ mutant egg chambers perform exactly one extra round of mitosis. (C-E) Serial confocal microscopy sections of this particular $d D P^{a 2}$ mutant egg chamber with double the appropriate number of nurse cells revealed five ring canals on the oocyte. Each arrow indicates a different ring canal. $12 \mathrm{~mm}$ separate $\mathrm{C}$ and E. (F) Projection composite of $11(2 \mathrm{~mm})$ serial sections of the egg chamber shown in (C-E). $d D P^{v r 10}$ and $d D P^{a 2}$, respectively; Table 1), whereas none of the unrescued eggs hatched. To determine whether the extra germ cell division phenotype was rescued, ovaries were dissected from mosaic females and the number of germ cells/egg chamber was determined by staining with DAPI and phalloidin. Only $5 \%$ of the $d D P^{a 2}$ clonal egg chambers containing the $d D P$ transgene had too many nurse cells, whereas $12 \%$ of egg chambers dissected from sibling flies lacking the transgene had twice the normal number of nurse cells (Table 2). Thus the two major phenotypes that we report for mutation of $\mathrm{dDP}$ in the female germline, i.e. a loss of dorsal-ventral polarity and a failure to correctly form a 16-cell cyst, are significantly rescued by a functional $d D P$ transgene.

\section{DISCUSSION}

By analyzing clones of mutant cells in the female germline we have determined that the DP subunit of the E2F transcription factor is required during Drosophila oogenesis. All phenotypes were observed with two independently isolated mutant alleles of $d D P$. These phenotypes include an inability to terminate germ cell division on schedule, a failure to transfer nurse cell cytoplasm to the oocyte, and disruption of dorsal-ventral polarity in developing egg chambers. The latter phenotypes were surprising, and reveal that certain E2F/DP complexes
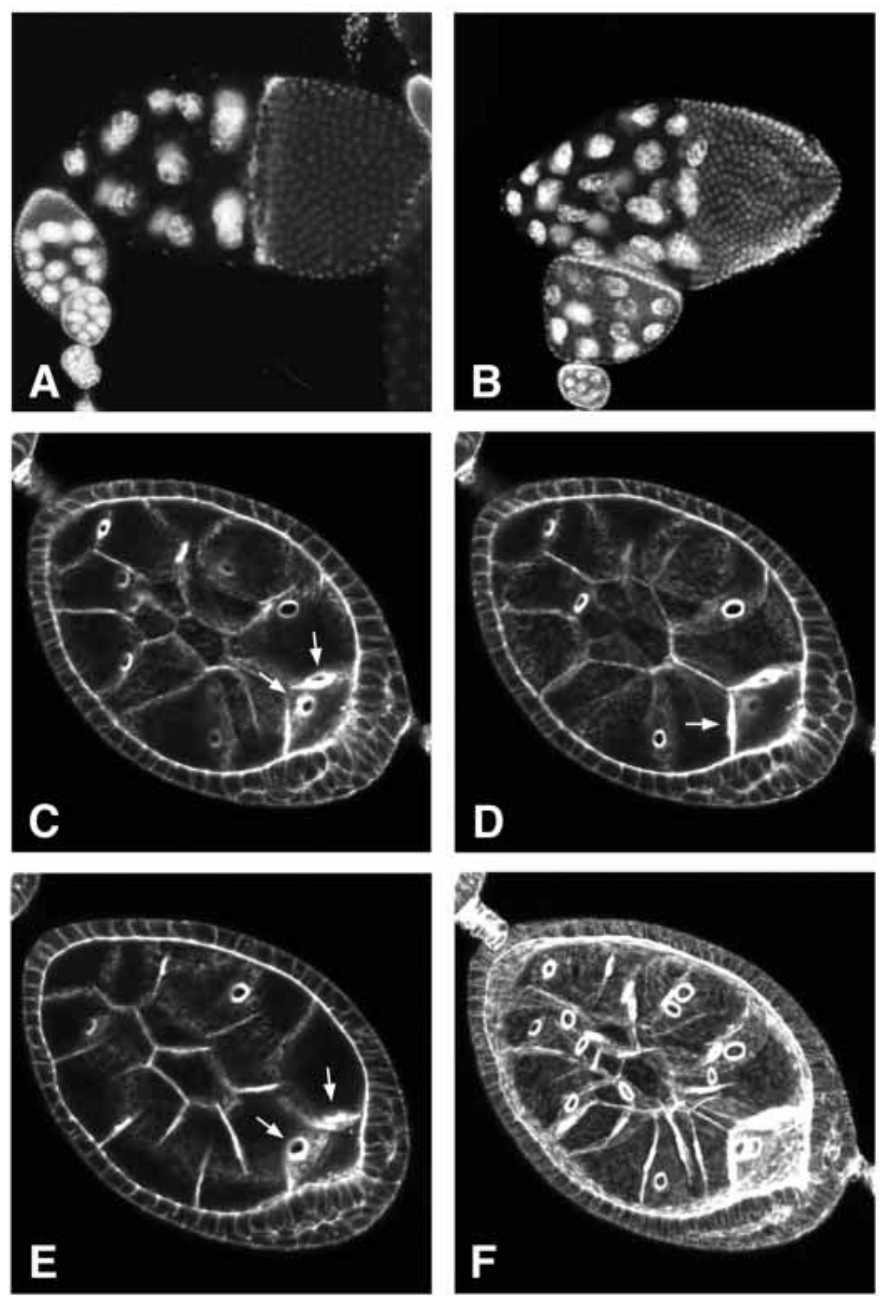
may contribute to important developmental processes, in addition to playing a role in cell cycle control.

Drosophila encodes two E2Fs (dE2F and dE2F2) that can each bind dDP (Dynlacht et al., 1994; Sawado et al., 1998), and that are each expressed maternally (Duronio et al., 1995) and P. Cayirlioglu and R. J. Duronio, unpublished). Mutation of the single $d D P$ gene should therefore eliminate the function of both $\mathrm{dE} 2 \mathrm{~F} / \mathrm{dDP}$ and $\mathrm{dE} 2 \mathrm{~F} 2 / \mathrm{dDP}$ heterodimers. Consequently, any or all of the $d D P$ germline phenotypes could be caused by disrupting one of these two complexes, or by disrupting both complexes simultaneously. Phenotypic characterization of $d E 2 F$ and $d E 2 F 2$ mutations in the female germline could distinguish between these possibilities. Clonal analysis of $d E 2 F$ in the germline may, however, be precluded by the inability of $d E 2 F$ mutant cells to proliferate (Neufeld et al., 1998), and $d E 2 F 2$ mutations have not yet been described. In contrast, analysis of the Drosophila $\mathrm{pRb}$ proteins has provided a clue. Unlike $d D P$, eggs laid by females lacking $R B F$ in the germline develop normally (Du and Dyson, 1999). Thus, loss of $\mathrm{RBF} / \mathrm{dE} 2 \mathrm{~F} / \mathrm{dDP}$ or $\mathrm{RBF} / \mathrm{dE} 2 \mathrm{~F} 2 / \mathrm{dDP}$ complexes does not readily explain the $d D P$ germline phenotypes. Interestingly, another gene $(R B F 2)$ with significant sequence similarity to the $\mathrm{pRb}$ family was revealed by the BDGP/Celera genome sequencing project. $R B F 2$ encodes a protein that interacts with $\mathrm{dDP}$ in coimmunoprecipitation experiments (O. Stevaux and N. Dyson, personal communication). This raises the possibility that $\mathrm{dDP}$ complexes containing RBF2 regulate the processes discussed here.

\section{The function of dDP in the germline cell cycle}

E2F/DP transcription factors are generally thought to drive cell cycle progression by activating the expression of genes required for growth and DNA synthesis. Our analysis, however, indicates that loss of $d D P$ function does not cause cell cycle arrest in the female germline. We readily detected clonal expansion of a founding $d D P$ mutant daughter cell in adult female ovaries approximately 2 weeks after mitotic recombination was induced in late larval stages. Furthermore, $d D P$ mutant nurse cell nuclei are capable of endoreduplication and become polyploid. Thus, in the germline dDP does not play an essential role in DNA synthesis, neither as a transcriptional activator nor as a direct contributor to the assembly of competent prereplicative complexes, as it does in the follicle cells (Asano and Wharton, 1999; Royzman et al., 1999). While these data are inconsistent with general models of E2F function, they are consistent with recent genetic data from Drosophila indicating that $\mathrm{dE} 2 \mathrm{~F} / \mathrm{dDP}$ dependent activation of transcription is not an absolute requirement for cell cycle progression (Du, 1999; Duronio et al., 1998; Royzman et al., 1997). For instance, $d D P$ null mutant progeny continue cell division and can survive until late in pupal development (Royzman et al., 1997). Removal of dDP should eliminate both transcriptional activation by cellular E2F/DP complexes and transcriptional repression by $\mathrm{RBF} / \mathrm{E} 2 \mathrm{~F} / \mathrm{DP}$ complexes. Accordingly, derepression of E2F target genes may sufficiently compensate for the lack of transcriptional activation at the $\mathrm{G}_{1}-\mathrm{S}$ transition to support cell cycle progression.

Rather than playing a strictly positive cell cycle role, our results indicate that $\mathrm{dDP}$ contributes to cell cycle arrest in the germline. In some egg chambers $d D P$ mutant germ cells failed to exit the cell cycle on schedule and entered an additional mitosis. In addition, (Royzman et al., 1999) have recently shown that $d D P$ is required for follicle cells to exit the endoreduplication cycle at the appropriate time. While the basis for these phenotypes is not presently known, we hypothesize that they represent a role for $\mathrm{dDP}$ in transcriptional repression in conjunction with an RBF family member. This is also supported by the observation that the $d M C M 3$ gene is derepressed in the CNS of $d D P$ mutant embryos (Duronio et al., 1998).

\section{The function of dDP in nurse cell cytoplasmic 'dumping'}

Drosophila oogenesis relies on communication between the nurse cells and the oocyte that includes transfer of nurse cell cytoplasm to the oocyte. There are two, successive phases of cytoplasmic transport during oogenesis. Early transport is slow and highly selective. Later in oogenesis a rapid phase of cytoplasmic transfer occurs as nurse cells contract to squeeze their contents into the oocyte. $d D P$ mutant egg chambers are defective in this rapid transport. They are also defective in nurse cell apoptosis. How might dDP affect both nurse cell cytoplasmic transport and apoptosis? Recent evidence reveals that entry into apoptosis may be coupled to rapid cytoplasm transport. In wild-type egg chambers several events indicate an apparent initiation of a nurse cell apoptotic pathway just prior to rapid cytoplasm transfer. The nurse cells undergo actin cytoskeleton rearrangements (Cooley et al., 1992; Gutzeit, 1986) and their nuclear membranes become permeabilized (Giorgi and Deri, 1976; McCall and Steller, 1998; Okada and Waddington, 1959). Delays in apoptosis seen in dumpless mutants that disrupt the actin cytoskeleton, such as chickadee (profilin) and kelch (a ring canal component), suggest that an apoptotic pathway and a rapid cytoplasmic transport pathway are interconnected (Foley and Cooley, 1998). Germline mutant clones of Drosophila dcp-1, a CED-3-related caspase (Song et al., 1997), display defects in actin bundle assembly, nuclear envelope permeabilization and cytoplasmic transport (McCall and Steller, 1998). Thus, Matova et al. (1999) have suggested that activation of an apoptotic pathway in nurse cells may lead to the formation of the actin bundles and subsequent nurse cell to oocyte cytoplasmic transfer. This model places a signal to enter apoptosis at the start of the pathway, followed by $d c p-1$ function, followed by chickadee, quail (villin) and singed (fascin) functions in bundling actin, and ending with cytoplasm transfer. In the context of this model, dDP could act either by directly regulating genes involved in actin cytoskeleton function (e.g. chickadee) or an apoptotic pathway (e.g. dcp-1). The well-documented ability of both mammalian and Drosophila E2F to induce apoptosis (Yamasaki, 1999) suggests the latter hypothesis, i.e. that $\mathrm{dDP}$ is required for the initiation of nurse cell apoptosis, which triggers subsequent cytoplasmic dumping.

\section{The function of dDP in dorsal-ventral patterning during oogenesis}

Surprisingly, loss of dDP function significantly inhibited the establishment of dorsal-ventral polarity during oogenesis. Germline cells mutant for either of two alleles of $d D P$ prevented the normal accumulation of Grk protein. In $d D P^{a 2}$ oocytes Grk protein failed to accumulate to wild-type levels. 
Grk protein was detected at higher levels in $d D P^{v r 10}$ mutants than $d D P^{a 2}$ mutants, but failed to properly localize to the oocyte plasma membrane. These data suggest that both Grk accumulation and intracellular localization are disrupted by mutation of $d D P$. Because Grk is required for an oocytefollicle cell signaling system that initiates dorsal follicle cell fate, the $d D P$ mutants displayed a ventralized eggshell.

One way that $\mathrm{dDP}$ could contribute to Grk accumulation is via transcriptional control of a target gene involved in this process. Several genes that affect the localized accumulation of Grk protein have been described (Ghabrial et al., 1998; Hawkins et al., 1997; Roth et al., 1995; Styhler et al., 1998; Suzanne et al., 1999; Tinker et al., 1998; Tomancak et al., 1998; Wilson et al., 1996). One of these genes, encore, behaves very much like $d D P$ in that it functions in both establishment of dorsal-ventral polarity and normal cell cycle control in the female germline. The encore gene is required for correct formation of the 16-cell cyst; encore mutants execute an additional mitotic cycle, such that 31 nurse cells and one oocyte are formed (Hawkins et al., 1996). encore also has an independent role in regulating Grk expression. encore mutants have reduced Grk levels and defects in dorsal-ventral polarity (Hawkins et al., 1997). How Encore regulates these processes is unclear, as little is known about its biochemical function. Regardless of the mechanism of Encore function, the similarity between the $d D P$ and encore mutant phenotypes suggests that $d D P$ may act upstream or in parallel to encore. In the simplest situation, dDP would regulate the expression of encore. We have analyzed Encore protein expression in $d D P$ mutant egg chambers and seen no difference compared to wild-type chambers (data not shown). Therefore we do not believe that dDP regulates encore.

Another possibility is that defects in Gurken accumulation result from disruption of the cell cycle caused by loss of $d D P$. There is already evidence that Gurken expression is linked to cell cycle control. Mutation of the 'spindle' class genes spnB and okra prevent normal Grk accumulation and establishment of the dorsal-ventral axis (Ghabrial et al., 1998; GonzalezReyes et al., 1997). spnB and okra encode proteins with sequence similarity to yeast genes required for repair of double strand breaks (DSBs) (Ghabrial et al., 1998). The failure to accumulate Grk in $\operatorname{spn} B$ and okra mutants is a downstream developmental consequence of a meiotic checkpoint activated in response to the persistence of DSB generated during meiotic recombination (Ghabrial and Schüpbach, 1999). Perhaps loss of $d D P$ function alters replication sufficiently to activate a checkpoint pathway. Alternatively, the dorsal-ventral defect in $d D P$ mutants may reflect a $\mathrm{dDP}$ requirement for expression of spnB and okra during meiosis.

Mutants in licorne, a Drosophila p38 mitogen-activated protein kinase kinase (MAPKK), have a highly penetrant dumpless phenotype in addition to a defect in dorsal-ventral polarity (Suzanne et al., 1999). The similarity of these phenotypes to those caused by mutation of $d D P$ that we report suggest a possible connection. While licorne could be a dDP target, it may instead act upstream of dDP. In mammalian cells the p38 kinase pathway can stimulate pRB phosphorylation, activation of E2F and the increase of E2F-mediated transcription (Wang et al., 1999). Thus, the p38 pathway may be required for $\mathrm{dE} 2 \mathrm{~F} / \mathrm{dDP}$ function in the female germline. It will be interesting and important to test these models.

\section{Conclusions}

Analysis of E2Fs in genetically tractable systems like Drosophila promises to reveal important functions for this transcription factor family. Here we have demonstrated a novel role for DP in establishing dorsal-ventral polarity during development. This discovery may represent an important need to coordinate the cell cycle with subsequent developmental events. Strikingly, a very recent paper has reported that E2F functions in the establishment of dorsal-ventral polarity in Xenopus embryos by regulating expression of ventral- and posterior-specific genes such as Hox genes (Suzuki and Hemmati-Brivanlou, 2000). Taken together, these observations suggest a possible evolutionarily conserved role for E2F transcription factors in pattern formation. It will be exciting to decipher which E2F/DP complexes are involved in particular developmental processes, as well as how these transcription factors may link cell cycle regulation and axis determination. These will be important steps toward fully understanding the many functions of this transcription factor family.

We thank Terry Orr-Weaver, Trudi Schüpbach and Nick Dyson for providing reagents and communicating results prior to publication. We thank Stephanie Hatch for helping make the $F R T, d D P$ recombinant chromosomes, Theresa Grana for hopping the $d D P$ transgene onto TM6, and Handan Kaygun for assistance in the analysis of $d D P^{v r 10}$ mutant eggs. Thanks also to Steve Crews, Jeff Sekelsky and Mark Peifer for comments on the manuscript, and a special thanks to Liz Gavis, Trudi Schüpbach and members of the Schüpbach laboratory for insight and helpful discussions. This work was supported in part by NIH postdoctoral training grant 5T3 CA09156 and NIH National Research Service Award to D.L.M. (1 F32 GM20019-01), NIH grant GM57859 to R.J.D., and the Cancer Research Fund of the Damon Runyon-Walter Winchell Foundation Award, DRS-10, to R.J.D.

\section{REFERENCES}

Asano, M. and Wharton, R. P. (1999). E2F mediates developmental and cell cycle regulation of ORC1 in Drosophila. EMBO J. 18, 2435-2448.

Brook, A., Xie, J. E., Du, W. and Dyson, N. (1996). Requirements for dE2F function in proliferating cells and in post-mitotic differentiating cells. EMBO J. 15, 3676-3683.

Cant, K., Knowles, B. A., Mooseker, M. S. and Cooley, L. (1994). Drosophila singed, a fascin homolog, is required for actin bundle formation during oogenesis and bristle extension. J. Cell Biol. 125, 369-380.

Chou, T. B., Noll, E. and Perrimon, N. (1993). Autosomal P[ovoD1] dominant female-sterile insertions in Drosophila and their use in generating germ-line chimeras. Development 119, 1359-1369.

Chou, T. B. and Perrimon, N. (1996). The autosomal FLP-DFS technique for generating germline mosaics in Drosophila melanogaster. Genetics 144, 1673-1679.

Cooley, L., Verheyen, E. and Ayers, K. (1992). chickadee encodes a profilin required for intercellular cytoplasm transport during Drosophila oogenesis. Cell 69, 173-184.

DeGregori, J., Leone, G., Miron, A., Jakoi, L. and Nevins, J. R. (1997) Distinct roles for E2F proteins in cell growth control and apoptosis. Proc. Natl. Acad. Sci. USA 94, 7245-7250.

Du, W. (1999). Suppression of the rbf null mutants by a de $2 \mathrm{f} 1$ allele that lacks transactivation domain. Development 127, 367-379.

Du, W. and Dyson, N. (1999). The role of RBF in the introduction of $\mathrm{G}_{1}$ regulation during Drosophila embryogenesis. EMBO J. 18, 916-925.

Du, W., Vidal, M., Xie, J. E. and Dyson, N. (1996). RBF, a novel RB-related gene that regulates E2F activity and interacts with cyclin E in Drosophila. Genes Dev. 10, 1206-1218.

Duronio, R. J., Bonnette, P. C. and O'Farrell, P. H. (1998). Mutations of the Drosophila dDP, dE2F, and cyclin E genes reveal distinct roles for the 
E2F-DP transcription factor and cyclin E during the $\mathrm{G}_{1}-\mathrm{S}$ transition. Mol. Cell. Biol. 18, 141-151.

Duronio, R. J. and O'Farrell, P. H. (1994). Developmental control of a $\mathrm{G}_{1-}$ S transcriptional program in Drosophila. Development 120, 1503-1515.

Duronio, R. J. and O'Farrell, P. H. (1995). Developmental control of the $\mathrm{G}_{1}$ to $\mathrm{S}$ transition in Drosophila: cyclin $\mathrm{E}$ is a limiting downstream target of E2F. Genes Dev. 9, 1456-1468.

Duronio, R. J., O'Farrell, P. H., Xie, J. E., Brook, A. and Dyson, N. (1995). The transcription factor E2F is required for S phase during Drosophila embryogenesis. Genes Dev. 9, 1445-1455.

Dynlacht, B. D., Brook, A., Dembski, M., Yenush, L. and Dyson, N. (1994). DNA-binding and trans-activation properties of Drosophila E2F and DP proteins. Proc. Natl. Acad. Sci. USA 91, 6359-6363.

Dyson, N. (1998). The regulation of E2F by pRB-family proteins. Genes Dev. 12, 2245-2262.

Ephrussi, A., Dickinson, L. K. and Lehmann, R. (1991). Oskar organizes the germ plasm and directs localization of the posterior determinant nanos. Cell 66, 37-50.

Field, S. J., Tsai, F. Y., Kuo, F., Zubiaga, A. M., Kaelin, W. G., Jr., Livingston, D. M., Orkin, S. H. and Greenberg, M. E. (1996). E2F-1 functions in mice to promote apoptosis and suppress proliferation. Cell $\mathbf{8 5}$, 549-561.

Foley, K. and Cooley, L. (1998). Apoptosis in late-stage Drosophila nurse cells does not require genes within the H99 deficiency. Development 125, 1075-1082.

Ghabrial, A., Ray, R. P. and Schüpbach, T. (1998). okra and spindle-B encode components of the RAD52 DNA repair pathway and affect meiosis and patterning in Drosophila oogenesis. Genes Dev. 12, 2711-2723.

Ghabrial, A. and Schüpbach, T. (1999). Activation of a meiotic checkpoint regulates translation of Gurken during Drosophila oogenesis. Nature Cell Biol. 1, 354-357.

Gilbert, S. F. (1994). Developmental Biology. Sunderland, MA: Sinauer Associates, Inc.

Giorgi, F. and Deri, P. (1976). Cell death in ovarian chambers of Drosophila melanogaster. J. Embryol. Exp. Morph. 35, 521-533.

Gonzalez-Reyes, A., Elliott, H. and St Johnston, D. (1995). Polarization of both major body axes in Drosophila by gurken-torpedo signalling. Nature 375, 654-658.

Gonzalez-Reyes, A., Elliott, H. and St Johnston, D. (1997). Oocyte determination and the origin of polarity in Drosophila: the role of the spindle genes. Development 124, 4927-4937.

Gutzeit, H. O. (1986). The role of microfilaments in cytoplasmic streaming in Drosophila follicles. J. Cell Sci. 80, 159-169.

Gutzeit, H. O. and Straub, A. (1989). Follicle cell development is partly independent of germ-line cell differentiation in Drosophila oogenesis. Roux's Arch. Dev. Biol. 198, 185-190.

Hao, X. F., Alphey, L., Bandara, L. R., Lam, E. W., Glover, D. and La Thangue, N. B. (1995). Functional conservation of the cell cycle-regulating transcription factor DRTF1/E2F and its pathway of control in Drosophila melanogaster. J. Cell Sci. 108, 2945-2954.

Harbour, J. W., Luo, R. X., Dei Santi, A., Postigo, A. A. and Dean, D. C. (1999). Cdk phosphorylation triggers sequential intramolecular interactions that progressively block $\mathrm{Rb}$ functions as cells move through $\mathrm{G}_{1}$. Cell $\mathbf{9 8}$, 859-869.

Hawkins, N. C., Thorpe, J. and Schüpbach, T. (1996). Encore, a gene required for the regulation of germ line mitosis and oocyte differentiation during Drosophila oogenesis. Development 122, 281-290.

Hawkins, N. C., Van Buskirk, C., Grossniklaus, U. and Schüpbach, T. (1997). Post-transcriptional regulation of gurken by encore is required for axis determination in Drosophila. Development 124, 4801-4810.

Humbert, P. O., Verona, R., Trimarchi, J. M., Rogers, C., Dandapani, S. and Lees, J. A. (2000). E2f3 is critical for normal cellular proliferation. Genes Dev. 14, 690-703.

Jordan, P. and Karess, R. (1997). Myosin light chain-activating phosphorylation sites are required for oogenesis in Drosophila. J. Cell Biol. 139, $1805-1819$.

Kim-Ha, J., Smith, J. L. and Macdonald, P. M. (1991). oskar mRNA is localized to the posterior pole of the Drosophila oocyte. Cell 66, 23-35.

Lasko, P. F. and Pardue, M. L. (1988). Studies of the genetic organization of the vestigial microregion of Drosophila melanogaster. Genetics 120, 495502 .

Lilly, M. A. and Spradling, A. C. (1996). The Drosophila endocycle is controlled by Cyclin E and lacks a checkpoint ensuring S-phase completion. Genes Dev. 10, 2514-2526.
Lindeman, G. J., Dagnino, L., Gaubatz, S., Xu, Y., Bronson, R. T., Warren, H. B. and Livingston, D. M. (1998). A specific, nonproliferative role for E2F-5 in choroid plexus function revealed by gene targeting. Genes Dev. 12, 1092-1098.

Mahajan-Miklos, S. and Cooley, L. (1994). The villin-like protein encoded by the Drosophila quail gene is required for actin bundle assembly during oogenesis. Cell 78, 291-301.

Manseau, L., Calley, J. and Phan, H. (1996). Profilin is required for posterior patterning of the Drosophila oocyte. Development 122, 2109-2116.

Matova, N., Mahajan-Miklos, S., Mooseker, M. S. and Cooley, L. (1999). Drosophila Quail, a villin-related protein, bundles actin filaments in apoptotic nurse cells. Development 126, 5645-5657.

McCaffrey, J., Yamasaki, L., Dyson, N. J., Harlow, E. and Griep, A. E. (1999). Disruption of retinoblastoma protein family function by human papillomavirus type $16 \mathrm{E} 7$ oncoprotein inhibits lens development in part through E2F-1. Mol. Cell. Biol. 19, 6458-6468.

McCall, K. and Steller, H. (1998). Requirement for DCP-1 caspase during Drosophila oogenesis. Science 279, 230-234.

Neufeld, T. P., de la Cruz, A. F., Johnston, L. A. and Edgar, B. A. (1998). Coordination of growth and cell division in the Drosophila wing. Cell 93, 1183-1193.

Neuman-Silberberg, F. S. and Schüpbach, T. (1993). The Drosophila dorsoventral patterning gene gurken produces a dorsally localized RNA and encodes a TGF alpha-like protein. Cell 75, 165-174.

Neuman-Silberberg, F. S. and Schüpbach, T. (1996). The Drosophila TGFalpha-like protein Gurken: expression and cellular localization during Drosophila oogenesis. Mech. Dev. 59, 105-113.

Ohtani, K. and Nevins, J. R. (1994). Functional properties of a Drosophila homolog of the E2F1 gene. Mol. Cell. Biol. 14, 1603-1612.

Okada, E. and Waddington, C. H. (1959). The submicroscopic structure of the Drosophila egg. J. Embryol. Exp. Morph. 7, 583-597.

Pan, H., Yin, C., Dyson, N. J., Harlow, E., Yamasaki, L. and Van Dyke, T. (1998). Key roles for E2F1 in signaling p53-dependent apoptosis and in cell division within developing tumors. Mol. Cell. 2, 283-292.

Persengiev, S. P., Kondova, II and Kilpatrick, D. L. (1999). E2F4 actively promotes the initiation and maintenance of nerve growth factor-induced cell differentiation. Mol. Cell. Biol. 19, 6048-6056.

Ray, R. P. and Schüpbach, T. (1996). Intercellular signaling and the polarization of body axes during Drosophila oogenesis. Genes Dev. 10, 1711-1723.

Rittenhouse, K. R. and Berg, C. A. (1995). Mutations in the Drosophila gene bullwinkle cause the formation of abnormal eggshell structures and bicaudal embryos. Development 121, 3023-3033.

Robinson, D. N. and Cooley, L. (1997a). Drosophila kelch is an oligomeric ring canal actin organizer. J. Cell Biol. 138, 799-810.

Robinson, D. N. and Cooley, L. (1997b). Genetic analysis of the actin cytoskeleton in the Drosophila ovary. Annu. Rev. Cell. Dev. Biol. 13, 147-170.

Robinson, D. N., Smith-Leiker, T. A., Sokol, N. S., Hudson, A. M. and Cooley, L. (1997). Formation of the Drosophila ovarian ring canal inner rim depends on cheerio. Genetics 145, 1063-1072.

Roth, S., Neuman-Silberberg, F. S., Barcelo, G. and Schüpbach, T. (1995). cornichon and the EGF receptor signaling process are necessary for both anterior-posterior and dorsal-ventral pattern formation in Drosophila. Cell 81, 967-978.

Royzman, I., Austin, R. J., Bosco, G., Bell, S. P. and Orr-Weaver, T. L. (1999). ORC localization in Drosophila follicle cells and the effects of mutations in dE2F and dDP. Genes Dev. 13, 827-840.

Royzman, I., Whittaker, A. J. and Orr-Weaver, T. L. (1997). Mutations in Drosophila DP and E2F distinguish $\mathrm{G}_{1}-\mathrm{S}$ progression from an associated transcriptional program. Genes Dev. 11, 1999-2011.

Sawado, T., Yamaguchi, M., Nishimoto, Y., Ohno, K., Sakaguchi, K. and Matsukage, A. (1998). dE2F2, a novel E2F-family transcription factor in Drosophila melanogaster. Biochem. Biophys. Res. Commun. 251, 409415

Schüpbach, T. (1987). Germ line and soma cooperate during oogenesis to establish the dorsoventral pattern of egg shell and embryo in Drosophila melanogaster. Cell 49, 699-707.

Schüpbach, T. and Wieschaus, E. (1991). Female sterile mutations on the second chromosome of Drosophila melanogaster. II. Mutations blocking oogenesis or altering egg morphology. Genetics 129, 1119-1136.

Song, Z., McCall, K. and Steller, H. (1997). DCP-1, a Drosophila cell death protease essential for development. Science 275, 536-540.

Spradling, A. C. (1993). Developmental genetics of oogenesis. In The Development of Drosophila melanogaster, vol. 1 (ed. Michael Bate and 
Alfonso Martinez Arias), pp. 1-70. Cold Spring Harbor, NY: Cold Spring Harbor Laboratory Press.

Styhler, S., Nakamura, A., Swan, A., Suter, B. and Lasko, P. (1998). vasa is required for GURKEN accumulation in the oocyte, and is involved in oocyte differentiation and germline cyst development. Development 125, 1569-1578.

Suzanne, M., Irie, K., Glise, B., Agnes, F., Mori, E., Matsumoto, K. and Noselli, S. (1999). The Drosophila p38 MAPK pathway is required during oogenesis for egg asymmetric development. Genes Dev. 13, 1464-1474.

Suzuki, E. and Hemmati-Brivanlou, A. (2000). Xenopus embryonic E2F is required for the formation of ventral and posterior cell fates during early embryogensis. Mol. Cell 5, 217-229.

Tautz, D. and Pfeifle, C. (1989). A non-radioactive in situ hybridization method for the localization of specific RNAs in Drosophila embryos reveals translational control of the segmentation gene hunchback. Chromosoma $\mathbf{9 8}$, $81-85$.

Tinker, R., Silver, D. and Montell, D. J. (1998). Requirement for the vasa RNA helicase in gurken mRNA localization. Dev. Biol. 199, 1-10.

Tomancak, P., Guichet, A., Zavorszky, P. and Ephrussi, A. (1998). Oocyte polarity depends on regulation of gurken by Vasa. Development 125, 17231732.

Tsai, K. Y., Hu, Y., Macleod, K. F., Crowley, D., Yamasaki, L. and Jacks, T. (1998). Mutation of E2f-1 suppresses apoptosis and inappropriate $S$ phase entry and extends survival of Rb-deficient mouse embryos. Mol. Cell 2, 293304.

Vigo, E., Muller, H., Prosperini, E., Hateboer, G., Cartwright, P., Moroni, M. C. and Helin, K. (1999). CDC25A phosphatase is a target of E2F and is required for efficient E2F- induced S phase. Mol. Cell. Biol. 19, 6379-6395.
Wang, S., Nath, N., Minden, A. and Chellappan, S. (1999). Regulation of $\mathrm{Rb}$ and E2F by signal transduction cascades: divergent effects of JNK1 and p38 kinases. EMBO J. 18, 1559-1570.

Wilson, J. E., Connell, J. E. and Macdonald, P. M. (1996). aubergine enhances oskar translation in the Drosophila ovary. Development 122, 16311639.

Xue, F. and Cooley, L. (1993). kelch encodes a component of intercellular bridges in Drosophila egg chambers. Cell 72, 681-693.

Yamaguchi, M., Hayashi, Y., Hirose, F., Nishimoto, Y. and Matsukage, A. (1997). Distinct roles of E2F recognition sites as positive or negative elements in regulation of the DNA polymerase alpha $180 \mathrm{kDa}$ catalytic subunit gene promoter during Drosophila development. Nucleic Acids Res. 25, 3847-3854.

Yamaguchi, M., Hayashi, Y. and Matsukage, A. (1995). Essential role of E2F recognition sites in regulation of the proliferating cell nuclear antigen gene promoter during Drosophila development. J. Biol. Chem. 270, 2515925165.

Yamasaki, L. (1999). Balancing proliferation and apoptosis in vivo: the Goldilocks theory of E2F/DP action. Biochim. Biophys. Acta 1423, M9-15.

Yamasaki, L., Bronson, R., Williams, B. O., Dyson, N. J., Harlow, E. and Jacks, T. (1998). Loss of E2F-1 reduces tumorigenesis and extends the lifespan of Rb1(+/-)mice. Nature Genet. 18, 360-364.

Yamasaki, L., Jacks, T., Bronson, R., Goillot, E., Harlow, E. and Dyson, N. J. (1996). Tumor induction and tissue atrophy in mice lacking E2F-1. Cell 85, 537-548.

Zheng, N., Fraenkel, E., Pabo, C. O. and Pavletich, N. P. (1999). Structural basis of DNA recognition by the heterodimeric cell cycle transcription factor E2F-DP. Genes Dev. 13, 666-674. 